\title{
Phase transfer and DNA functionalization of quantum dots using an easy to prepare, low-cost zwitterionic polymer
}

\author{
Margaret Chern ${ }^{1}$ \\ Chloe Grazon ${ }^{2,3}$ \\ Allison Dennis ${ }^{1,4, *}$
}

${ }^{1}$ Division of Materials Science and Engineering, Boston University, 15 St. Mary’s Street, Boston, MA

${ }^{2}$ Department of Chemistry, Boston University, 590 Commonwealth Ave, Boston, MA

${ }^{3}$ Univ. Bordeaux, CNRS, Bordeaux INP, LCPO, UMR 5629, F-33600, Pessac, France

${ }^{4}$ Department of Biomedical Engineering, Boston University, 44 Cummington Mall, Boston, MA

Running Head: Decorating zwitterionic quantum dots with oligomeric DNA 


\section{i. Phase transfer and DNA functionalization of quantum dots using an easy to prepare, low-cost zwitterionic polymer}

\section{ii. Summary/Abstract}

Small, stable, and bright quantum dots (QDs) are of interest in many biosensing and biomedical imaging applications, but current methodologies for obtaining these characteristics can be highly specialized or expensive. We describe a straightforward, low-cost protocol for functionalizing poly(isobutylene-alt-maleic anhydride) (PIMA) with moieties that anchor to the QD surface (histamine), impart hydrophilicity ((2-aminoethyl)trimethylammonium chloride $\left.\left(\mathrm{Me}_{3} \mathrm{~N}^{+}-\mathrm{NH}_{2}\right)\right)$, and provide a platform for biofunctionalization via click chemistry (dibenzocyclooctyne (DBCO)). Guidelines to successfully use this polymer for QD ligand exchange are presented and an example of biofunctionalization with DNA is shown. Stable QD-DNA conjugates are obtained with high yield and without requiring additional purification steps.

\section{iii. Key Words}

Quantum dots, zwitterion, DNA labeling, surface functionalization, copper-free click chemistry, biofunctionalization, nanoparticle, carboxybetaine, poly(isobutylene-alt-maleic anhydride), PIMA

\section{Introduction}

Phase Transfer and DNA Functionalization of Quantum Dots Using an Easy-to-Prepare, Low-Cost Zwitterionic Polymer 
Quantum dots (QDs) are semiconductor nanocrystals that exhibit size-tunable fluorescence that is bright, narrow, and photostable. This can be advantageous in biological applications that require high signal to noise or long-term exposure to light. However, the majority of quantum dots are synthesized in organic solutions and must be post-processed to impart water solubility. The methods for achieving this are vast and varied.(1-3) Commercially available, water-soluble QDs often employ an amphiphilic polymer that encapsulates the QD with its original organic ligands. While quite stable, their large hydrodynamic size can negatively affect energy transfer $(4,5)$ and the robustness of the steric coating precludes the use of histidine-based selfassembly of peptides and proteins to the QD, the simplest way to make QD bioconjugates.(5, 6) Ligand exchange strategies that displace the native organic ligands create smaller particles. The first iterations of this method used small molecule ligands, but reports have shown that increasing the number of chemical handles to bind to the QD surface improves stability. $(7,8)$ Building on that knowledge, several groups have reported the synthesis of multidentate polymers(9-11) for QD water-solubilization that provide excellent colloidal stability. The Mattoussi group has developed a particularly compelling strategy using a poly(anhydride maleicalt-isobutylene) (PIMA) as a polymer backbone for facile functionalization with primary amines.(12) They have shown effective grafting of amine bearing imidazole, lipoic acid, and phosphonates for anchoring the polymer to the QD surface, as well as sulfobetaine and polyethylene glycol (PEG) derivatives for water solubilization.(13-15) Interest in zwitterionic or charge-based surface coatings, like the PIMA variant displaying sulfobetaine, is high as the surface charges are reported to confer excellent colloidal stability and reduce protein adsorption or opsonization.(16) Unfortunately, synthesizing and handling sulfobetaine or a similar Zwitterionic molecule carboxybetaine with a primary amine is non-trivial, as is the synthesis of Phase Transfer and DNA Functionalization of Quantum Dots Using an Easy-to-Prepare, Low-Cost Zwitterionic Polymer M. Chern, C. Grazon, A. M. Dennis Published in Quantum Dots - Applications in Biology Springer Protocols 2020 
zwitterionic polymers from zwitterionic monomers. To expand access to those who are not organic chemists, we outline a straightforward protocol for preparing a low-cost, charged polymer (3.1) and using it in a straightfoward ligand exchange (3.2) to obtain an aqueous colloidal solution of bright, small, and stable QDs.(17)

Rather than using polyethylene glycol (PEG; expensive) or sulfobetaine (difficult to synthesize) derivatives to functionalize the PIMA, we use a commercially available quaternary amine ((2aminoethyl)trimethylammonium chloride $\left.\left(\mathrm{Me}_{3} \mathrm{~N}^{+}-\mathrm{NH}_{2}\right)\right)$ to impart a persistent positive charge. Combined with the carboxylic acid produced on each PIMA moiety formed during the amide coupling between the maleic anhydride and the primary amine-containing molecules, the $\mathrm{Me}_{3} \mathrm{~N}^{+}$$\mathrm{NH}_{2}$ produces a carboxybetaine-like zwitterionic coating without the need to synthesize a zwitterionic agent. Histamine, used as the anchoring group mediating the interaction between the QD surface and the polymer, is also readily available. To make the polymer relevant to a variety of biological applications, we include dibenzocyclooctyne-amine (DBCO-amine), which can react with any azido biomolecule of interest (e.g., derivatives of ligands like folic acid or biotin as well as larger peptides or proteins). Specifically, we detail our purification-free protocol for grafting DNA to the QD surface (3.3). Research in creating QD-DNA conjugates is ongoing, and historically, methods have been low-yield with the highest grafting efficiencies reaching only $\sim 65 \%(9,18)$ necessitating downstream purification steps.(19) The protocol we provide is simple and highly efficient ( $>95 \%$ ) precluding the need for purification and increasing the overall yield of the QD-DNA conjugates obtained.

\section{Materials}

Phase Transfer and DNA Functionalization of Quantum Dots Using an Easy-to-Prepare, Low-Cost Zwitterionic Polymer M. Chern, C. Grazon, A. M. Dennis

Published in Quantum Dots - Applications in Biology Springer Protocols 2020 


\subsection{Polymer Synthesis, Purification, and Characterization}

1. Polymer backbone: Poly(isobutylene-alt-maleic anhydride) (PIMA; molecular weight $(\mathrm{MW}) \sim 6,000 \mathrm{~g} / \mathrm{mol})$. PIMA has been largely shelf-stable in our experience, but since water can hydrolyze the rings needed for functionalization, long-term storage in a desiccator or in a glove box at room temperature is preferred.

2. Primary amine-containing molecules to functionalize polymer: (2-aminoethyl) trimethylammonium chloride hydrochloride $\left(\mathrm{Me}_{3} \mathrm{~N}^{+}-\mathrm{NH}_{2}\right)$ and triethylamine $\left(\mathrm{Et}_{3} \mathrm{~N}\right)$ for solubilization; histamine; dibenzocyclooctyne-amine (DBCO- $\left.\mathrm{NH}_{2}\right)$ (see Note 1).

3. Anhydrous dimethylsulfoxide (DMSO) (see Note 2).

4. Polymer precipitation solution: Pre-weigh two $50 \mathrm{~mL}$ conical tubes, taking care to mark which lid belongs to which tube. Prepare $50 \mathrm{~mL}$ of ethyl acetate/diethyl ether (1/1) by mixing $25 \mathrm{~mL}$ of ethyl acetate and $25 \mathrm{~mL}$ diethyl ether and divide between the two tubes. Mixing the ethyl acetate and diethyl ether in one of the tubes and pouring half into the other is precise enough for this purpose and ensures that the solvent ratio is the same in both tubes.

5. Methanol $(\mathrm{MeOH})$.

6. Deuterium oxide $\left(\mathrm{D}_{2} \mathrm{O}\right)$.

\subsection{Quantum Dot Preparation and Ligand Exchange}

1. Quantum dots (see Note 3).

2. Organic solvents: chloroform $\left(\mathrm{CHCl}_{3}\right)$, hexanes (Hex), methanol $(\mathrm{MeOH})$, ethanol (EtOH) (see Note 4).

Optional:

Phase Transfer and DNA Functionalization of Quantum Dots Using an Easy-to-Prepare, Low-Cost Zwitterionic Polymer 
- $\quad 0.2 \mathrm{M}$ zinc stearate in octadecene (ODE): weigh $6.3233 \mathrm{~g}$ of zinc stearate and load into a $100 \mathrm{~mL}$ round bottom flask. Add a stir bar. Heat while stirring under vacuum to $80^{\circ} \mathrm{C}$ until fully dissolved. Once dissolved, fill the flask with nitrogen and stir for 15 mins before switching back to vacuum and degassing for 15 mins. Do at least 3 vacuum / nitrogen cycles to ensure that the solution is air-free.

- $\quad 0.1 \mathrm{M}$ sodium bicarbonate $\left(\mathrm{NaHCO}_{3}\right)$ : mix $8.401 \mathrm{~g}$ of $\mathrm{NaHCO}_{3}$ with $1 \mathrm{~L} \mathrm{diH}_{2} 0$.

- $\quad 0.1 \mathrm{M}$ sodium hydroxide $(\mathrm{NaOH})$.

\subsection{DNA Labeling and Characterization}

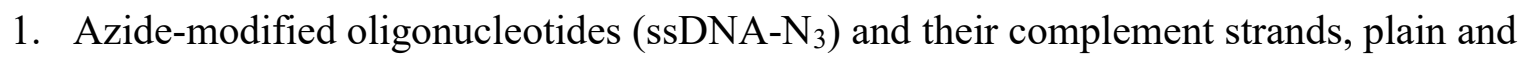
biotinylated (csDNA \& csDNA-bt), can be custom ordered, for example from IDT Technologies).

2. 1 x Duplex Buffer: $100 \mathrm{mM}$ Potassium Acetate, $30 \mathrm{mM}$ HEPES, $\mathrm{pH} 7.5$, available from IDT Technologies.

3. Streptavidin-coated agarose beads.

4. $2 \mathrm{M}$ sodium chloride $(2 \mathrm{M} \mathrm{NaCl})$ : Add $1.17 \mathrm{~g}$ of $\mathrm{NaCl}$ to graduated cylinder and raise the volume to $10 \mathrm{~mL}$ with $\mathrm{diH}_{2} \mathrm{O}$. Add stir bar and leave on a stir plate until completely dissolved ( 1 $\mathrm{min})$.

\section{Methods}

\section{$\underline{3.1 \text { Polymer Preparation }}$}

Phase Transfer and DNA Functionalization of Quantum Dots Using an Easy-to-Prepare, Low-Cost Zwitterionic Polymer 
All synthesis steps should be carried out under air-free conditions

(https://www.chemistryviews.org/details/education/4360441/Tips and Tricks for the Lab_Air-

Sensitive Techniques_3.html). Either nitrogen or argon can be used as the inert gas in this protocol.

This procedure is specific to preparing a polymer for ligand exchange that is functionalized with $45 \%$ histamine, $45 \% \mathrm{Me}_{3} \mathrm{~N}^{+}-\mathrm{NH}_{2}$, and $10 \% \mathrm{DBCO}-\mathrm{NH}_{2}$ (Scheme 1). Different grafting percentages can be obtained by adjusting the molar ratios of each reagent. Additional or different functional groups can be included as long as they contain a primary amine $\left(\mathrm{R}-\mathrm{NH}_{2}\right)$. It can be convenient to prepare a table (Table 1) for calculating the amount of each reagent needed. For PIMA with $\mathrm{MW} \sim 6,000 \mathrm{~g} / \mathrm{mol}$ there are on average $\sim 39$ monomers $(\mathrm{MW}=154 \mathrm{~g} / \mathrm{mol}$ ) per polymer. We calculate our molar equivalents by assuming 40 monomers/polymer in order to provide slight excess of amine bearing reagents.

\section{Prepare Solution 1:}

a. Weigh $0.240 \mathrm{~g}$ of PIMA into a $25 \mathrm{~mL}$ round bottom flask equipped with a magnetic stir bar and sealed with a septum.

b. Insert a needle (see Note 5) in the septum and degas either by $i$ ) actively flowing nitrogen through the flask (using a second needle to allow nitrogen to escape, creating flow) while gently stirring the powder for 20 min or ii) applying 3 cycles of vacuum / nitrogen for 5 seconds each (see Note 6).

c. Add $6 \mathrm{~mL}$ of anhydrous DMSO to the dry powder with a syringe. The PIMA should dissolve easily in DMSO.

Phase Transfer and DNA Functionalization of Quantum Dots Using an Easy-to-Prepare, Low-Cost Zwitterionic Polymer 
d. Heat Solution 1 to $45{ }^{\circ} \mathrm{C}$ while stirring.

2. Prepare Solution 2:

a. In a separate septum-sealed container (glass vial or round bottom flask), combine histamine (80 mg), $\mathrm{Me}_{3} \mathrm{~N}^{+}-\mathrm{NH}_{2}(126 \mathrm{mg})$, and $\mathrm{DBCO}-\mathrm{NH}_{2}(44 \mathrm{mg})$ at 18, 18, and 4 molar equivalents to PIMA, respectively. Include a magnetic stir bar.

b. Add $\mathrm{Et}_{3} \mathrm{~N}$ at a 2:1 molar ratio to $\mathrm{Me}_{3} \mathrm{~N}-\mathrm{NH}_{2}(200 \mu \mathrm{L})$ to help solubilize $\mathrm{Me}_{3} \mathrm{~N}^{+}$in DMSO.

c. Insert a needle in the septum and degas by either $i$ ) actively flowing nitrogen through the flask while gently stirring the mixture for 5 min or ii) applying 3 cycles of vacuum / nitrogen for 5 seconds each. Be careful not to evaporate the triethylamine.

d. Add anhydrous DMSO with a syringe such that the final concentration of all reagents in solution is $260 \mathrm{mM}(11.5 \mathrm{~mL})$.

e. Heat Solution 2 to $50{ }^{\circ} \mathrm{C}$ while stirring to fully dissolve reagents.

3. Add Solution 2 to Solution 1 (stirring at $45^{\circ} \mathrm{C}$ ) dropwise with a syringe.

4. Allow the mixture to stir overnight at $45^{\circ} \mathrm{C}$.

\subsubsection{Polymer Purification and Characterization}

1. Add the polymer/DMSO solution (18 mL total) dropwise to the two pre-weighed $50 \mathrm{~mL}$ conical tubes each containing $\sim 25 \mathrm{~mL}$ of precipitating solvent $(\sim 9 \mathrm{~mL}$ polymer solution in each tube). The solution will become turbid (cloudy), indicating that the polymer is precipitating.

Phase Transfer and DNA Functionalization of Quantum Dots Using an Easy-to-Prepare, Low-Cost Zwitterionic Polymer 
2. Centrifuge the $50 \mathrm{~mL}$ conical tube ( 7,000 RPM, $5-15 \mathrm{mins})$ to obtain a pellet of the PIMA polymer (see Note 7). Discard the supernatant containing unreacted amines and triethylamine.

3. Set the conical tubes upside down on a laboratory wipe and let sit for $\sim 5$ mins to dry the pellet.

4. Add $\mathrm{MeOH}(\sim 1-5 \mathrm{~mL})$ directly to each tube to re-disperse the polymer pellet (see Note 8).

5. Precipitate the polymer from $\mathrm{MeOH}$ by adding ethyl acetate until the solution becomes turbid $(\sim 10-20 \mathrm{~mL})$.

6. Centrifuge to obtain a pellet of the purified polymer and discard the supernatant.

7. Dry the purified polymer under vacuum. Septa $(24 / 40$ or $29 / 32)$ can be used to seal the 50 $\mathrm{mL}$ conical vials and attached to the vacuum/gas manifold with a syringe and needle. Alternatively, a vacuum desiccator can be used, if available.

8. The mass of the polymer is obtained by weighing the tube with polymer and subtracting the original weight of the tube. The chemical yield is estimated by dividing the end weight by the original total mass of the reaction. The yield should be higher than $70 \%$.

9. After purification and drying, a white powder is obtained and should be stored at $4{ }^{\circ} \mathrm{C}$ in the dark for further use.

10. Acquire an infrared spectrum of the powder using an attenuated total reflection (ATR) probe on a Fourier-transform infrared (FTIR) spectrophotometer to confirm the disappearance of the $\mathrm{C}=\mathrm{O}$ stretch of the anhydride at $1770 \mathrm{~cm}^{-1}$ and appearance of the $\mathrm{C}=\mathrm{O}$ stretch of carboxylic acid and amide at $1710 \mathrm{~cm}^{-1}$ and $1650 \mathrm{~cm}^{-1}$, respectively

\section{(Figure 1).}

Phase Transfer and DNA Functionalization of Quantum Dots Using an Easy-to-Prepare, Low-Cost Zwitterionic Polymer 
11. Run ${ }^{1} \mathrm{H}$ NMR in $\mathrm{D}_{2} \mathrm{O}$ to confirm the purity of the polymer and its molar composition (example calculations provided in Figure 2; see Note 9).

\subsection{Quantum Dot (QD) Preparation and Phase Transfer}

1. Aliquot $\sim 150 \mu \mathrm{L}$ of QDs in organic solution into a $2 \mathrm{~mL}$ centrifuge tube and add $\sim 150 \mu \mathrm{L}$ of hexanes. Commercially sourced QDs ship at $\sim 1 \mu \mathrm{M}$. The QDs used in this procedure started at $4.8 \mu \mathrm{M}$.

2. Add EtOH until the solution becomes cloudy.

3. Centrifuge to obtain a pellet of the "cleaned" QDs (see Notes 10 \& 11).

4. Re-disperse the QDs in $\mathrm{CHCl}_{3}$ to a final concentration of $\sim 100 \mathrm{nM}(7 \mathrm{~mL})$.

5. Syringe filter $(0.1 \mu \mathrm{m}, \mathrm{PVDF})$ and transfer the $1 \mathrm{~mL}$ of the QDs in $\mathrm{CHCl}_{3}$ to a glass vial with a stir bar (see Notes 12 \& 13).

6. Prepare ligand solution: Weigh polymer in a glass vial and add anhydrous DMSO such that the final concentration is $10 \mathrm{mg} / \mathrm{mL}$. Use $\sim 10 \mathrm{mg}$ of polymer per $\mathrm{nmol}$ of QD ( 800:1 molar excess of polymer) (see Note 14). Heating with a heat gun or at $50{ }^{\circ} \mathrm{C}$ in an oil or water bath is usually required to fully dissolve the polymer.

7. Add the ligand solution dropwise to the QD solution while stirring at room temperature. For $1 \mathrm{~mL}$ of QDs at $100 \mathrm{nM}, 100 \mu \mathrm{L}$ of polymer at $10 \mathrm{mg} / \mathrm{mL}$ should be added. The final solution should consist of 10:1 $\mathrm{CHCl}_{3}$ :DMSO.

8. Purge the vial with nitrogen and allow the solution to stir for at least 1 hour at room temperature and protected from light (foil wrapped) (see Note 15).

9. For transfer to water, add $0.5 \mathrm{~mL}$ of $0.1 \mathrm{M} \mathrm{NaOH}$ to the QD/PIMA/DMSO/CHCl 3 mixture while stirring (see Note 16).

Phase Transfer and DNA Functionalization of Quantum Dots Using an Easy-to-Prepare, Low-Cost Zwitterionic Polymer 
10. Turn off stir plate and allow the aqueous phase and organic phases to separate. Successful ligand exchange is indicated by QDs being in the top (aqueous) phase (Figure 3). (see Notes 17-19)

11. Transfer to a centrifuge tube and centrifuge at $3000 \mathrm{rpm}$ for $1 \mathrm{~min}$ to obtain two nicely separated phases (Figure 3) and precipitate any aggregates. Separate the supernatant and collect the top, aqueous layer containing QDs in $0.1 \mathrm{M} \mathrm{NaOH}$.

12. Filter the QDs in $0.1 \mathrm{M} \mathrm{NaOH}$ on a $0.1 \mu \mathrm{m}$ (or $0.25 \mu \mathrm{m}$ ) PVDF syringe filter into a 100 $\mathrm{kDa}$ centrifugal filter ( $0.5 \mathrm{~mL}$ capacity) (see Note 20$)$.

13. Wash the QDs with $0.5 \mathrm{~mL}$ of $0.1 \mathrm{M} \mathrm{NaHCO}_{3}$ and centrifuge at $10,000 \mathrm{rpm}$ for $2 \mathrm{~min}$.

14. Discard the flow-through and refill the centrifugal filter with $0.1 \mathrm{M} \mathrm{NaHCO}_{3}$.

15. Centrifuge at $10,000 \mathrm{rpm}$ for 2 mins.

16. Repeat steps 14 and 15 at least two more times.

17. Recover the QDs in a minimal amount of buffer (now in $0.1 \mathrm{M} \mathrm{NaHCO}_{3}$ ) and store concentrated at $4^{\circ} \mathrm{C}$ in the dark.

\subsection{DNA Functionalization}

\subsubsection{DBCO stoichiometry determination}

1. The number of DBCO moieties per QD can be estimated with UV-Vis absorbance. DBCO absorbs strongly in the UV and has a molar extinction coefficient $(\varepsilon)$ of $12,000 \mathrm{M}^{-}$ ${ }^{1} \mathrm{~cm}^{-1}$ at $309 \mathrm{~nm}$. Scale QD absorbance before ligand exchange to the absorbance spectra of the QD after ligand exchange at $400 \mathrm{~nm}$. Calculate QD concentration using the BeerLambert Law and the QD molar extinction coefficient given by the vendor or using published size-dependent molar extinction coefficient formulas.(20)

Phase Transfer and DNA Functionalization of Quantum Dots Using an Easy-to-Prepare, Low-Cost Zwitterionic Polymer 
Beer-Lambert Law:

$$
A_{\lambda}=\varepsilon_{\lambda} \cdot c \cdot l
$$

where $A \lambda$ is absorbance at wavelength $\lambda, \varepsilon$ is molar extinction coefficient $\left(\mathrm{M}^{-1} \mathrm{~cm}^{-1}\right)$ at wavelength $\lambda, c$ is concentration $(\mathrm{M})$, and $l$ is pathlength $(\mathrm{cm})$.

2. Subtract the absorbance before ligand exchange from absorbance after ligand exchange to obtain DBCO absorbance. Use the value of the absorbance at $309 \mathrm{~nm}$ to calculate DBCO concentration using the Beer-Lambert Law.

3. Divide DBCO concentration by QD concentration to obtain $\mathrm{DBCO} / \mathrm{QD}$ ratio (Figure 4). The DNA grafting reaction relies on DBCO-azide coupling, so the $\mathrm{DBCO} / \mathrm{QD}$ ratio can be used to ensure that there are a sufficient amount of biofunctional handles on the QD.

\subsubsection{DNA conjugation and hybridization}

1. Add PIMA-coated QDs $(0.2 \mathrm{nmol} ; 70 \mu \mathrm{L}$ at $\sim 3 \mu \mathrm{M})$ and DNA-N $\mathrm{D}_{3}(4 \mathrm{nmol} ; 20 \mu \mathrm{L}$ at $200 \mu \mathrm{M}$ ) to a $500 \mu \mathrm{L}$ PCR tube at a DNA:QD ratio of 20:1(see Note 21).

2. Add $\mathrm{NaCl}$ to the QD/DNA mixture to obtain a final $\mathrm{NaCl}$ concentration of $1 \mathrm{M}(90 \mu \mathrm{L}$ of $2 \mathrm{M} \mathrm{NaCl})$.

3. Wrap the tube in aluminum foil and shake on an agitation plate for 4 days at room temperature. 
4. Centrifuge with a table top centrifuge for $\sim 1$ min to pellet any aggregates (see Note 22). Collect the supernatant.

\subsubsection{DNA hybridization:}

1. Add 4 nmol (i.e., an equimolar amount) of the DNA complement to the QD/ssDNA conjugates (see Note 23).

2. Heat to $95{ }^{\circ} \mathrm{C}$ for 2 minutes and gradually cool. A cooling ramp can be used, but simply transferring samples from a heat block/water batch to bench-top at room temperature is usually sufficient (see Note 24).

3. The QD-dsDNA can now be stored at $4{ }^{\circ} \mathrm{C}$ in the dark for further use. Do not freeze.

\subsubsection{Verification of hybridization:}

1. For simple verification of hybridization, a small aliquot of the QD-DNA should be hybridized with a biotinylated complement (available from IDT) (QD-dsDNA-bt), and hybridization can be verified in a simple bead pull-down assay.

2. Aliquot $\sim 200 \mu \mathrm{L}$ of streptavidin-agarose beads into a $500 \mu \mathrm{L}$ PCR tube.

3. Centrifuge for $30 \mathrm{~s}$ using a tabletop centrifuge $(\sim 6,000 \mathrm{rpm}, 2,000 \mathrm{~g})$ to pellet beads at the bottom of the tube.

4. Pull off the supernatant $(\sim 100 \mu \mathrm{L})$ with a pipette and add $100 \mu \mathrm{L}$ of $0.1 \mathrm{M} \mathrm{NaHCO}_{3}$. Pipette up and down 5 - 10 times to mix.

5. Repeat steps $3 \& 4$ two more times to fully wash the streptavidin beads.

6. Split bead slurry into two $500 \mu \mathrm{L}$ PCR tubes (100 $\mu \mathrm{L}$ each). 
7. To one tube (Tube 1) add 2-10 $\mu$ L of the QD-dsDNA. Pipette up and down $5-10$ times to mix.

8. To the other tube (Tube 2) add 2-10 $\mu \mathrm{L}$ of the QD-dsDNA-bt. Pipette up and down $5-10$ times to mix.

9. Centrifuge for $30 \mathrm{~s}$ using a tabletop centrifuge $(\sim 6,000 \mathrm{rpm}, 2,000 \mathrm{~g})$ to pellet beads at the bottom of the tube.

10. Repeat steps $3 \& 4$ two times to wash unbound QDs from the slurry.

11. Check for fluorescence under UV illumination (lamp, flashlight, etc.) The QD-dsDNA should not bind to the beads, so Tube 1 should be non-fluorescent, while the opposite is true of QD-dsDNA-bt (Figure 5).

\section{Notes}

1. Histamine and $\mathrm{DBCO}-\mathrm{NH}_{2}$ should be stored at $-20{ }^{\circ} \mathrm{C}$. When using reagents from the freezer, they should be taken out prior to the reaction and left to heat to room temperature before opening to avoid water condensation.

2. When taking anhydrous DMSO from a commercial septum-sealed bottle, make sure you use an argon or nitrogen balloon to compensate the volume of solvent taken with a syringe and needle. Do not use needles larger than 16-gauge to puncture through the septum.

3. This protocol can be used with QDs from a wide variety of sources, including in-house lab-made QDs of any number of compositions (e.g., CdSe, InP, etc.) capped with a ZnS shell or commercially available QDs sold in organic solvents (e.g., Qdot ${ }^{\mathrm{TM}} \mathrm{XXX}$ ITK $^{\mathrm{TM}}$

Phase Transfer and DNA Functionalization of Quantum Dots Using an Easy-to-Prepare, Low-Cost Zwitterionic Polymer 
Organic Quantum Dots sold by ThermoFisher Scientific, where the xxx denotes the peak emission wavelength of the QDs). The key QD characteristics critical to successfully encapsulation are a $\mathrm{ZnS}$ capping shell, as this passivation layer ensures that they are still photoluminescent once transferred into water. If using QDs synthesized in house, it is helpful to terminate the QD surface with cation (zinc) because the histamine (imidazole) has affinity to divalent cations.

4. Although many labs, including ours, have wash bottles of commonly used solvents available for convenience, we find that using ethanol freshly aliquoted from the reagent bottle has a significant effect on maintaining the brightness of the final QD product. Ethanol is hygroscopic and will draw water out of the air, gradually contaminating the solvent.

5. Consider the inner diameter of the tubing on the manifold and roughly match to the outer diameter of a plastic syringe, likely either the $1 \mathrm{~mL}$ or $3 \mathrm{~mL}$ size. Remove and discard the syringe plunger. Cut the syringe barrel just below the flanges on the back end to produce a plastic tube with a needle connection. Insert the straight barrel of the cut syringe into the manifold tubing; attach a needle and insert needle into septum.

6. If vacuum/inert gas cycling is used to degas the flasks relatively quickly, be sure to gradually switch from nitrogen to vacuum, as sudden introduction can cause the PIMA powder to fly about and potentially be drawn up the vacuum line..

7. If the polymer does not precipitate easily, place the conical tubes in the freezer $\left(-20^{\circ} \mathrm{C}\right)$ for $1 \mathrm{hr}$ and re-centrifuge. If a refrigerated centrifuge is available, centrifuge at $-20^{\circ} \mathrm{C}$.

8. If the polymer does not dissolve readily, gentle sonication, gentle heating (warning: boiling point of $\mathrm{MeOH}$ is $65^{\circ} \mathrm{C}$ ), or adding acetonitrile can help.

Phase Transfer and DNA Functionalization of Quantum Dots Using an Easy-to-Prepare, Low-Cost Zwitterionic Polymer 
9. If purification by precipitation does not yield high purity, the polymer can be further purified by dialysis ( $<10 \mathrm{kDa}$ membrane). This should be done after FTIR as any unreacted maleic anhydride rings will open in this process and confound FTIR results. Resuspend the product in a minimal amount of water and load into dialysis tubing or cassette. Place in a beaker filled with milli-Q water $(100-1,000 \mathrm{~mL})$; add a couple drops of $1 \mathrm{M} \mathrm{NaOH}$ to the dialysis bath to help swell the polymer and remove impurities. Let dialysis proceed for at least $1 \mathrm{hr}$ (longer is not harmful to the product, but will extend the time of the procedure). Replace the dialysis bath with milli-Q water and again allow the dialysis to proceed for at least one hour. The dialysis bath should be replaced at least 5 times with milli-Q water to remove all impurities.

10. Sometimes extra washing steps (re-dispersing in decane (or hexanes) and repeating step 2 in section 3.2) can help if the ligand transfer does not work for a specific QD sample. It is very important to remember to perform step 5 (section 3.2) if several QD precipitations are done because QDs will start to aggregate as more organic ligands are cleaned from their surface.

11. QDs that have not been successful in ligand transfer may benefit from a post-synthesis treatment with zinc stearate. After step 3 (section 3.2), the QD pellet can be re-dispersed directly in a small amount $(\sim 1 \mathrm{~mL})$ of zinc stearate dissolved in ODE $(0.2 \mathrm{M}$, heated to $\left.\sim 80^{\circ} \mathrm{C}\right)$. Transfer the $\mathrm{QD} / \mathrm{Zn}$ solution to a septum-fitted glass vial with a stir bar. Degas the solution by bubbling nitrogen through the solution. Heat while stirring at $240^{\circ} \mathrm{C}$ for 2 3 hours. The QDs should then be precipitated from solution by addition of hexane and ethanol and centrifuging. Extra washing steps are necessary when doing zinc treatment.

Phase Transfer and DNA Functionalization of Quantum Dots Using an Easy-to-Prepare, Low-Cost Zwitterionic Polymer 
Alternatively, the Smith group outlines several additional surface treatments and can be referred to for those who are more comfortable with chemistry.(9)

12. The rest of the QDs can be stored in a tightly sealed vial at $4{ }^{\circ} \mathrm{C}$ in the dark for up to a week or long term ( $\sim 2$ months $)$ at $-20^{\circ} \mathrm{C}$. Be sure to syringe filter and check the QD concentration by UV-vis. absorption the solution before ligand transfer.

13. For samples that are sensitive to oxidation, the vial can be septum capped and nitrogen gently bubbled through the solution ( $\sim 5$ mins $)$ while stirring. Dip the vial in an ice bag to avoid evaporation of the $\mathrm{CHCl}_{3}$.

14. If nanoparticle size is known, scaling to nanoparticle surface area instead can help to reduce waste of polymer. We find that $\sim 300$ anchoring groups (imidazoles) per $\mathrm{nm}^{2} \mathrm{QD}$ (QD concentration multiplied by the surface area of 1 QD) consistently works.

15. 1 hour is normally sufficient for ligand exchange to have occurred. However, overnight ligand exchange it has been qualitatively observed to impart better stability at the cost of decreased quantum yield.

16. Alternatively, the QDs can first be precipitated from the $\mathrm{DMSO} / \mathrm{CHCl}_{3}$ solution by addition of ethyl acetate and centrifugation $(10,000 \mathrm{rpm}, \sim 5 \mathrm{mins})$. The supernatant should then be discarded and the pellet recovered directly into $0.1 \mathrm{M} \mathrm{NaOH}$.

17. Sometimes a white precipitate is seen at the solvent interface. This can be pelleted through centrifugation and discarded.

18. If the phase transfer failed and QDs are in the bottom organic phase, they can be recollected and the ligand exchange re-attempted. The QD solution should be first filtered through a $0.1 \mu \mathrm{m}$ PVFD syringe filter to get rid of any aggregates that may have formed and a higher polymer/QD ratio should be used.

Phase Transfer and DNA Functionalization of Quantum Dots Using an Easy-to-Prepare, Low-Cost Zwitterionic Polymer 
19. If the QDs are stuck between the water and organic phases, sometimes they can be recovered. Remove the $\mathrm{CHCl}_{3} / \mathrm{DMSO}$ layer with a syringe and pour off the water layer + QDs into a $2 \mathrm{~mL}$ centrifuge tube. This can be gently sonicated to disperse the QDs in the water phase. Once relatively dispersed, hexanes can be added to the tube to wash off any excess organic ligands that may have gotten "stuck" between the polymer and QD during ligand exchange. The tube should then be sonicated ( $\sim 5$ mins) and centrifuged $(\sim 3,000$ rpm for $1 \mathrm{~min}$ ). QDs in the water phase (bottom) can be collected and used. If this does not work, the ligand transfer should be repeated from the beginning, using freshly prepared QDs in $\mathrm{CHCl}_{3}$ and a higher polymer ratio.

20. Sometimes at step 12 (section 3.2), the QDs are lost on the PVDF filter, indicating that large aggregates were solubilized rather than single QDs. Double check that the QD concentration in $\mathrm{CHCl}_{3}$ is $\sim 100 \mathrm{nM}$; if the solution is too concentrated, multiple QDs can be wrapped together during ligand exchange. Alternatively, increasing the $\mathrm{CHCl}_{3} \mathrm{DMSO}$ ratio by using a ligand solution at $20 \mathrm{mg} / \mathrm{mL}$ rather than $10 \mathrm{mg} / \mathrm{mL}$ can help to avoid QD aggregation during DMSO addition.

21. Higher or lower DNA/QD ratios can be used, but the DBCO/DNA ratio should be higher than 2:1

22. We have consistently seen that using the conditions described in the DNA conjugation methods provides near complete reaction efficiency of the DNA to the QDs (>95\%). Most downstream applications do not need further purification. For applications extremely sensitive to free DNA, dialysis or centrifugal filters can be used to separate QD-DNA conjugates from free DNA.

Phase Transfer and DNA Functionalization of Quantum Dots Using an Easy-to-Prepare, Low-Cost Zwitterionic Polymer 
23. High salt concentration can affect DNA hybridization. Double check your oligo's melting temperature at $1000 \mathrm{mM} \mathrm{NaCl}$ before proceeding with hybridization (https://www.idtdna.com/calc/analyzer/). If salt concentration needs to be lowered, the solution can be diluted with 1X duplex buffer (100 mM Potassium Acetate; $30 \mathrm{mM}$ HEPES, pH 7.5; available from IDT).

24. https://www.idtdna.com/pages/education/decoded/article/annealing-oligonucleotides can be referred to for more detailed instruction.

\section{References}

1. Medintz IL, Uyeda HT, Goldman ER, et al (2005) Quantum dot bioconjugates for imaging, labelling and sensing. Nat Mater 4:435-446

2. Karakoti AS, Shukla R, Shanker R, et al (2015) Surface functionalization of quantum dots for biological applications. Adv Colloid Interface Sci 215:28-45

3. Zhou J, Liu Y, Tang J, et al (2017) Surface ligands engineering of semiconductor quantum dots for chemosensory and biological applications. Mater Today 20:360-376

4. Chern M, Nguyen TT, Mahler AH, et al (2017) Shell thickness effects on quantum dot brightness and energy transfer. Nanoscale 9:16446-16458

5. Dennis AM, Sotto DC, Mei BC, et al (2010) Surface ligand effects on metal-affinity coordination to quantum dots: Implications for nanoprobe self-assembly. Bioconjug Chem $21: 1160-1170$

6. Medintz IL, Huston AL, Susumu K, et al (2013) Optimizing Protein Coordination to Quantum Dots with Designer Peptidyl Linkers. Bioconjug Chem 24:269-281

Phase Transfer and DNA Functionalization of Quantum Dots Using an Easy-to-Prepare, Low-Cost Zwitterionic Polymer 
7. Zhan N, Palui G, Safi M, et al (2013) Multidentate zwitterionic ligands provide compact and highly biocompatible quantum dots. J Am Chem Soc 135:13786-13795

8. Mattoussi H, Uyeda HTT, Medintz ILIL, et al (2005) Synthesis of compact multidentate ligands to prepare stable hydrophilic quantum dot fluorophores. J Am Chem Soc $127: 3870-3878$

9. Ma L, Tu C, Le P, et al (2016) Multidentate Polymer Coatings for Compact and Homogeneous Quantum Dots with Efficient Bioconjugation. J Am Chem Soc 138:33823394

10. Susumu K, Oh E, Delehanty JB, et al (2014) A new family of pyridine-appended multidentate polymers as hydrophilic surface ligands for preparing stable biocompatible quantum dots. Chem Mater 26:5327-5344

11. Wang W, Kapur A, Ji X, et al (2015) Photoligation of an Amphiphilic Polymer with Mixed Coordination Provides Compact and Reactive Quantum Dots. J Am Chem Soc $137: 5438-5451$

12. Mattoussi H and Wang W (2016), Multifunctional and multicoordinating amphiphilic polymer ligands for interfacing semiconducting, magnetic, and metallic nanocrystals with biological systems, https://patents.google.com/patent/US10040874B2/en

13. Du L, Wang W, Zhang C, et al (2018) A Versatile Coordinating Ligand for Coating Semiconductor, Metal, and Metal Oxide Nanocrystals. Chem Mater 30:7269-7279

14. Wang W, Aldeek F, Ji X, et al (2014) A multifunctional amphiphilic polymer as a platform for surface-functionalizing metallic and other inorganic nanostructures. Faraday Discuss 175:137-151

15. Wang W, Ji X, Kapur A, et al (2015) A Multifunctional Polymer Combining the Phase Transfer and DNA Functionalization of Quantum Dots Using an Easy-to-Prepare, Low-Cost Zwitterionic Polymer M. Chern, C. Grazon, A. M. Dennis

Published in Quantum Dots - Applications in Biology Springer Protocols 2020 
Imidazole and Zwitterion Motifs as a Biocompatible Compact Coating for Quantum Dots. J Am Chem Soc 137:14158-14172

16. Wang W, Kapur A, Ji X, et al (2016) Multifunctional and High Affinity Polymer Ligand that Provides Bio-Orthogonal Coating of Quantum Dots. Bioconjug Chem 27:2024-2036

17. Grazon C, Chern M, Ward K, et al (2019) A versatile and accessible polymer coating for functionalizable zwitterioinic quantum dots with high DNA grafting efficiency. Chem Commun In Submiss

18. Banerjee A, Grazon C, Nadal B, et al (2015) Fast, Efficient, and Stable Conjugation of Multiple DNA Strands on Colloidal Quantum Dots. Bioconjug Chem 26:1582-1589

19. Banerjee A, Pons T, Lequeux N, et al (2016) Quantum dots-DNA bioconjugates:

Synthesis to applications. Interface Focus 6:20160064

20. Reiss P, Protière M, and Li L (2009) Core/shell semiconductor nanocrystals. 5:154-168

Phase Transfer and DNA Functionalization of Quantum Dots Using an Easy-to-Prepare, Low-Cost Zwitterionic Polymer M. Chern, C. Grazon, A. M. Dennis

Published in Quantum Dots - Applications in Biology Springer Protocols 2020 


\section{Figures}
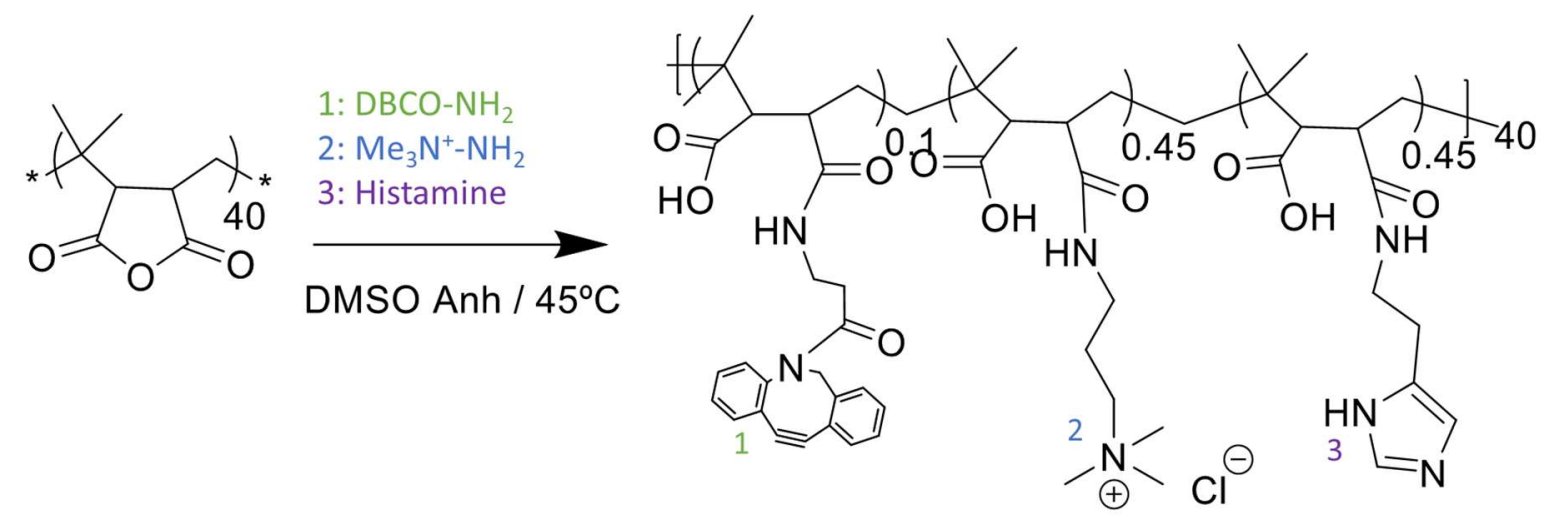

Scheme 1. Polymer Functionalization Scheme. Maleic anhydrides react with primary amines to functionalize the PIMA backbone. Hydrophilic or charged moieties are added in combination with anchoring groups for use as a coating to impart water solubility to QDs. Specifically, histamine is used to self-assemble the polymer to the QD surface, $\mathrm{Me}_{3} \mathrm{~N}^{+}$provides hydrophilicity and counter-balances the negative charges of carboxylic acids on the PIMA backbone, and DBCO enables further bioconjugation via copper-free click chemistry. 



Figure 1. FTIR spectra of the as-shipped (grey) and functionalized PIMA polymer (blue). The full spectrum is plotted on the left, while a narrower region is plotted on the right to highlight the wavelengths of interest.

Phase Transfer and DNA Functionalization of Quantum Dots Using an Easy-to-Prepare, Low-Cost Zwitterionic Polymer 


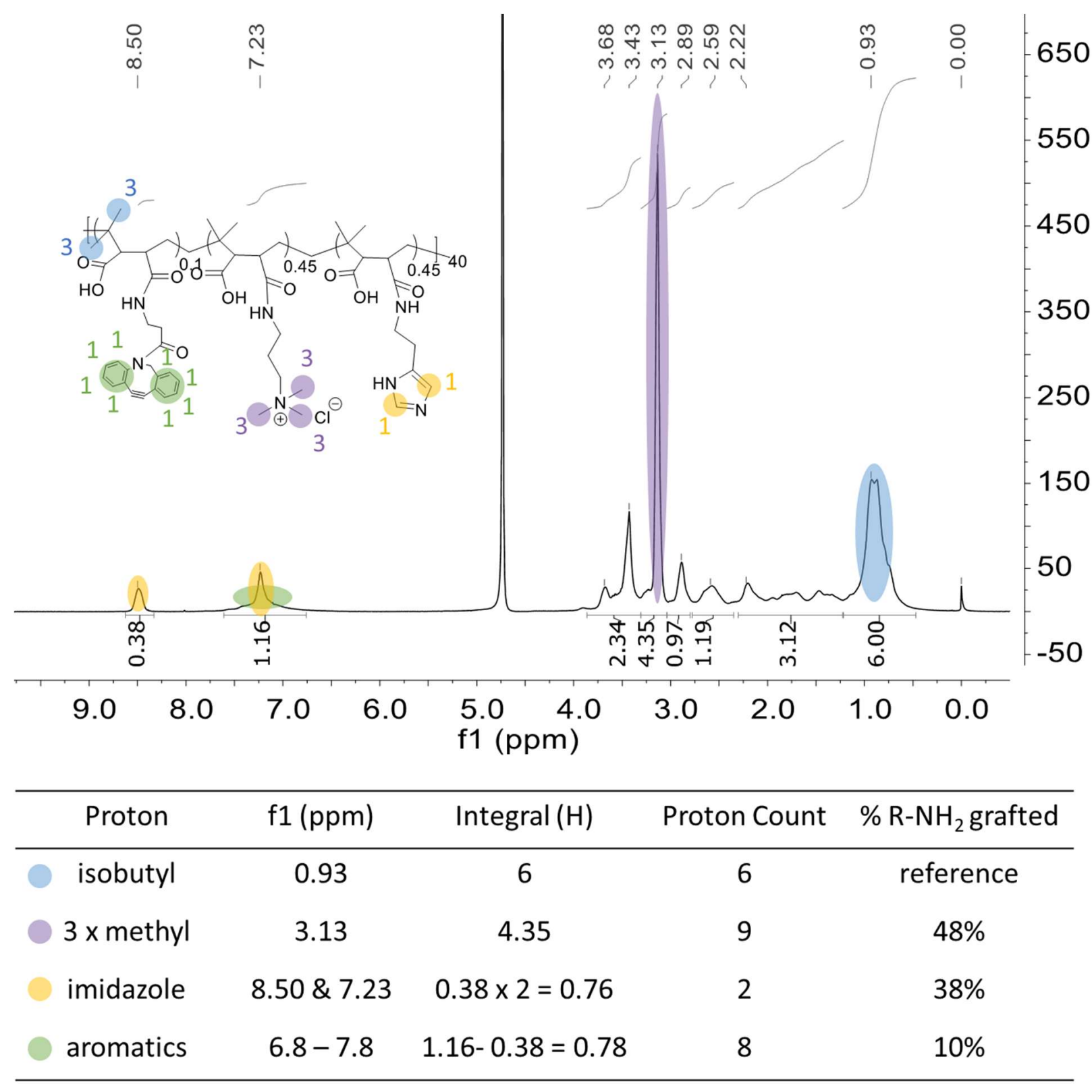

Figure 2. Analysis of ${ }^{1} \mathrm{H}$ NMR of the polymer in $\mathrm{D}_{2} \mathrm{O}$. The isobutyl group is used as reference (6 protons). Percentage of $\mathrm{R}^{-\mathrm{NH}_{2}}$ grafted is calculated by dividing the measured integral by each molecule's proton count. The $1 \mathrm{H}$ signal of the imidazole at 7.23 overlaps the $8 \mathrm{H}$ signal from the aromatics on the DBCO. For the imidazole, the $1 \mathrm{H}$ signal at 7.23 is estimated to be the same as that at 8.5 , so the $\mathrm{DBCO}$ integral is estimated by subtracting the integral from $6.8-7.8$ by that of the $1 \mathrm{H}$ from imidazole at 8.5 .

Phase Transfer and DNA Functionalization of Quantum Dots Using an Easy-to-Prepare, Low-Cost Zwitterionic Polymer 
Figure 3. Quantum dots ligand exchanged with the functionalized PIMA. Successful ligand exchange and phase transfer is indicated by the QDs moving from the bottom (organic) phase to the upper (water) phase.

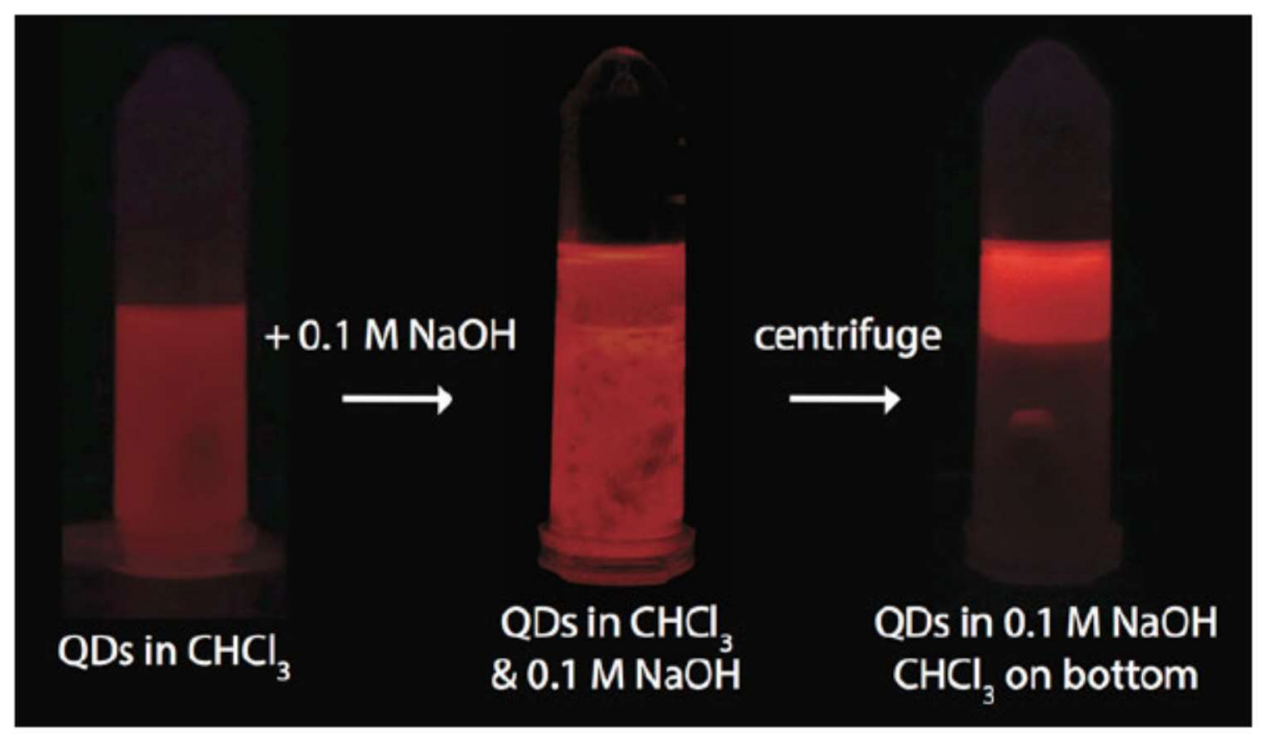

Figure 4. DBCO/QD Analysis. Absorbance spectra of the QDs in $\mathrm{CHCl}_{3}$ is normalized at $400 \mathrm{~nm}$ to the absorbance of the QDs coated with the functionalized PIMA ligand to obtain the contribution to absorbance from DBCO. The concentration of the QDs and DBCO can then be calculated separately and used to calculated $\mathrm{DBCO} / \mathrm{QD}$ ratio.

Phase Transfer and DNA Functionalization of Quantum Dots Using an Easy-to-Prepare, Low-Cost Zwitterionic Polymer 


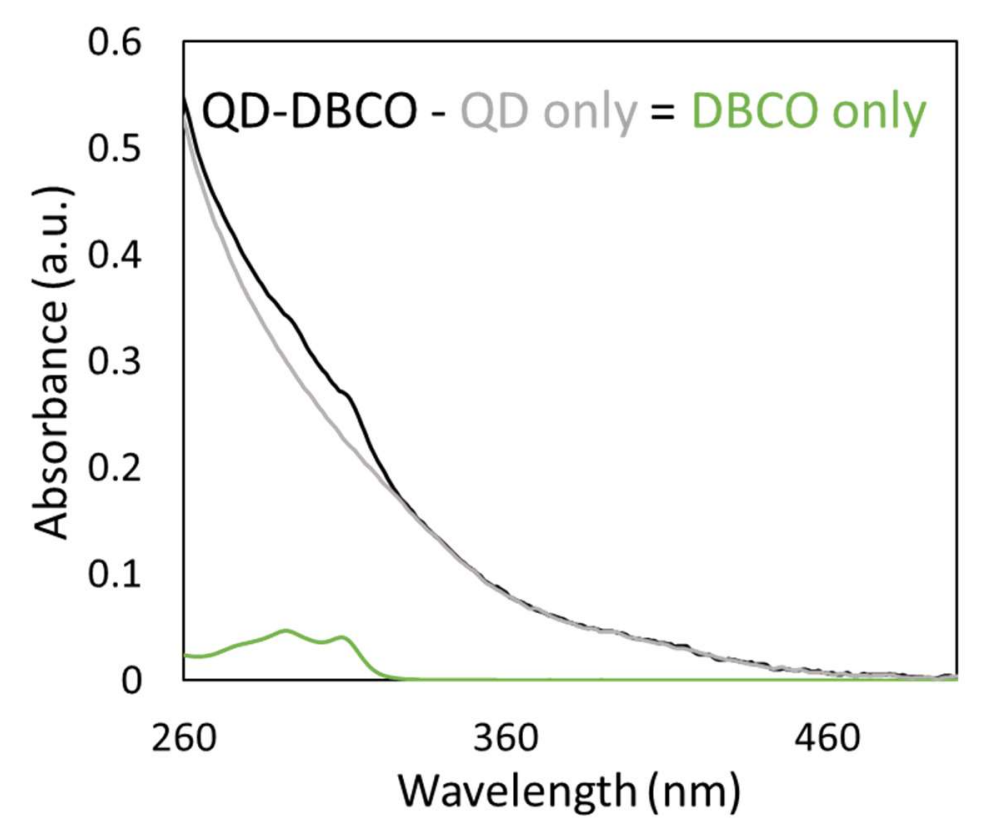

\begin{tabular}{ccc}
\hline & QD @ 400 nm & DBCO only @ 309 nm \\
\hline Absorbance & 0.04 & 0.04 \\
MEC $\left(\mathrm{M}^{-1} \mathrm{~cm}^{-1}\right)$ & $1.5 \times 10^{6}$ & $1.2 \times 10^{4}$ \\
pathlength $(\mathrm{cm})$ & 1 & 1 \\
Concentration $\left(\mathrm{M}^{-1}\right)$ & $2.7 \times 10^{-8}$ & $3.3 \times 10^{-6}$ \\
\hline \multicolumn{2}{l}{$\mathrm{DBCO} / \mathrm{QD}=3.3 \times 10^{-6} / 2.7 \times 10^{-8}=125$} \\
\hline
\end{tabular}

Figure 5. Verification of DNA hybridization. QDs hybridized with a plain complement strand (QD-dsDNA) (left tubes) do not have biotin and thus control for non-specific binding. QDs hybridized with a biotinylated complement strand (QD-dsDNA-bt) bind to the streptavidin beads, resulting in post-wash fluorescence (pink at bottom of tube) that indicates successful hybridization of the QD-bound DNA. 


\section{Table Captions:}

Table 1. Calculation of reagent amounts needed for a reaction consisting of $45 \%$ histamine, $45 \%$ $\mathrm{Me}_{3} \mathrm{~N}^{+}$and $10 \%$ DBCO- $\mathrm{NH}_{2} .{ }^{\mathrm{a}}$ Molar equivalents obtained by multiplying desired grafting fraction by the number of monomer/PIMA polymer $(\sim 40) .{ }^{b}$ Millimoles (mmol) of each reagent needed, calculated by multiplying $\mathrm{N}$ by the number of mmol of PIMA. PIMA is first weighed and the number of mmol calculated by dividing mass $(\mathrm{m})^{\mathbf{d}}$ in $\mathrm{mg}$ by $\mathrm{MW}^{\mathbf{c}}$.

\section{Tables:}

Table 1:

\begin{tabular}{|c|c|c|c|c|c|c|c|c|c|}
\hline Reagent & $\begin{array}{l}\text { Reagent } \\
\text { Fraction }\end{array}$ & $\begin{array}{l}\text { Molar } \\
\text { Equiv. }{ }^{a}\end{array}$ & $\begin{array}{l}\text { Moles }^{b} \\
\text { (mmol) }\end{array}$ & $\begin{array}{c}\mathrm{MW}^{c} \\
(\mathrm{~g} / \mathrm{mol})\end{array}$ & $\begin{array}{l}\text { Mass }^{d} \\
\text { (mg) }\end{array}$ & $\begin{array}{l}\text { Density } \\
(\mathrm{g} / \mathrm{mL})\end{array}$ & $\begin{array}{c}\text { Volume } \\
(\mu \mathrm{L})\end{array}$ & $\begin{array}{l}\text { [Final] } \\
(\mathrm{mM})\end{array}$ & $\begin{array}{c}\text { DMSO } \\
(\mathrm{mL})\end{array}$ \\
\hline PIMA & & 1 & 0.04 & 6000 & 240 & & & 6.7 & 6 \\
\hline histamine & 0.45 & 18 & 0.72 & 111.15 & 80 & & & 260 & 11.5 \\
\hline $\mathrm{Me}_{3} \mathrm{~N}^{+}-\mathrm{NH}_{2}$ & 0.45 & 18 & 0.72 & 175.10 & 126 & & & & \\
\hline${\mathrm{DBCO}-\mathrm{NH}_{2}}_{2}$ & 0.1 & 4 & 0.16 & 276.33 & 44 & & & & \\
\hline $\mathrm{Et}_{3} \mathrm{~N}$ & & 36 & 1.44 & 101.49 & 146 & 0.73 & 200 & & \\
\hline
\end{tabular}

Phase Transfer and DNA Functionalization of Quantum Dots Using an Easy-to-Prepare, Low-Cost Zwitterionic Polymer 